\title{
10-year Trends in non-operating Room Anesthesia Case Volumes at an American Academic Medical Center
}

\author{
Michael A Phelps MD and William D. Bradley MHA \\ Dept of Anesthesiology \& Critical Care Medicine \\ Johns Hopkins University (Baltimore, MD USA)
}

\begin{abstract}
Background and Goal of Study: Non-operating room anesthesia (NORA) represents a rapidly growing trend in anesthetic practice. Few studies, however, have quantified this trend, either overall or for subgroups of NORA.
\end{abstract}

Materials and Methods: With Institutional Review Board permission, we queried anesthesia billing records from July I, 2005 to June 30, 2015 at the Johns Hopkins Hospital which had billing labels identifying them as a NORA service. Each billed anesthetic was attempted categorization into a specific NORA service, such as gastrointestinal (Gl) endoscopy or interventional pulmonology (IP), using a combination of billing labels and Current Procedural Terminology (CPT) anesthesia descriptors. These billing records were subsequently analyzed to determine the NORA case volumes and trends over the 10 -year period studied.

Results and Discussion: A total of I347/8 NORA anesthetics were performed at the Johns Hopkins Hospital between July I, 2005 to June 30, 20I5. Of these, 6856 represented intubations performed in Intensive Care Units (ICU's) and medical floors throughout the hospital.

The total number of NORA cases increased by over $157 \%$ from the 2005-2006 academic year (July I, 2005 to June 30, 2006 ) to the 20I4-20I5 academic year (July I, 20I4 to June 30, 2015). The largest case volume increase over that timespan was from the Gl service, increasing from 3024 to 9725 cases per year from 2005-6 to 20I4-I5. This represents a $222 \%$ increase in that time period.

The greatest percentage increase were in cardiac electrophysiology cases, which climbed $2906 \%$ (52 cases in 2005-6 to 1563 cases in 2014-15). The next highest percentage increase was Interventional Pulmonology, up 681\%. Interventional Radiology cases increased by $272 \%$, electroconvulsive therapy increased by $58 \%$, and anesthesia for percutaneous tracheostomies in ICU's were up $226 \%$. Pediatric NORA volumes were relatively flat, declining slightly by II\%.

Conclusion(s): NORA case volume at the Johns Hopkins Hospital increased substantially over the 10 -year period from 2005 to 2015.
Annual NORA Cases By Service (2005-6 vs 20 I4- I 5 Academic Years)

\begin{tabular}{l|rrr} 
& $\mathbf{2 0 0 5 - 6}$ & $\mathbf{2 0 1 4 - 1 5}$ & \% Change \\
\hline Gastrointestinal Endoscopy & 3024 & 9725 & $222 \%$ \\
Interventional Radiology & 209 & 778 & $272 \%$ \\
Electroconvulsive Therapy & 1192 & 1890 & $59 \%$ \\
Pediatric NORA & 2153 & 1915 & $-11 \%$ \\
Interventional Pulmonology & 123 & 961 & $681 \%$ \\
Percutaneous Tracheostomies & 38 & 124 & $226 \%$ \\
Intubations & 155 & 810 & $423 \%$ \\
Cardiac Electrophysiology & 52 & 1563 & $2906 \%$ \\
Other NORA & 279 & 780 & $180 \%$ \\
Total NORA & 7225 & 18546 & $157 \%$
\end{tabular}

Total NORA Cases by Quarter (2005-20I5)

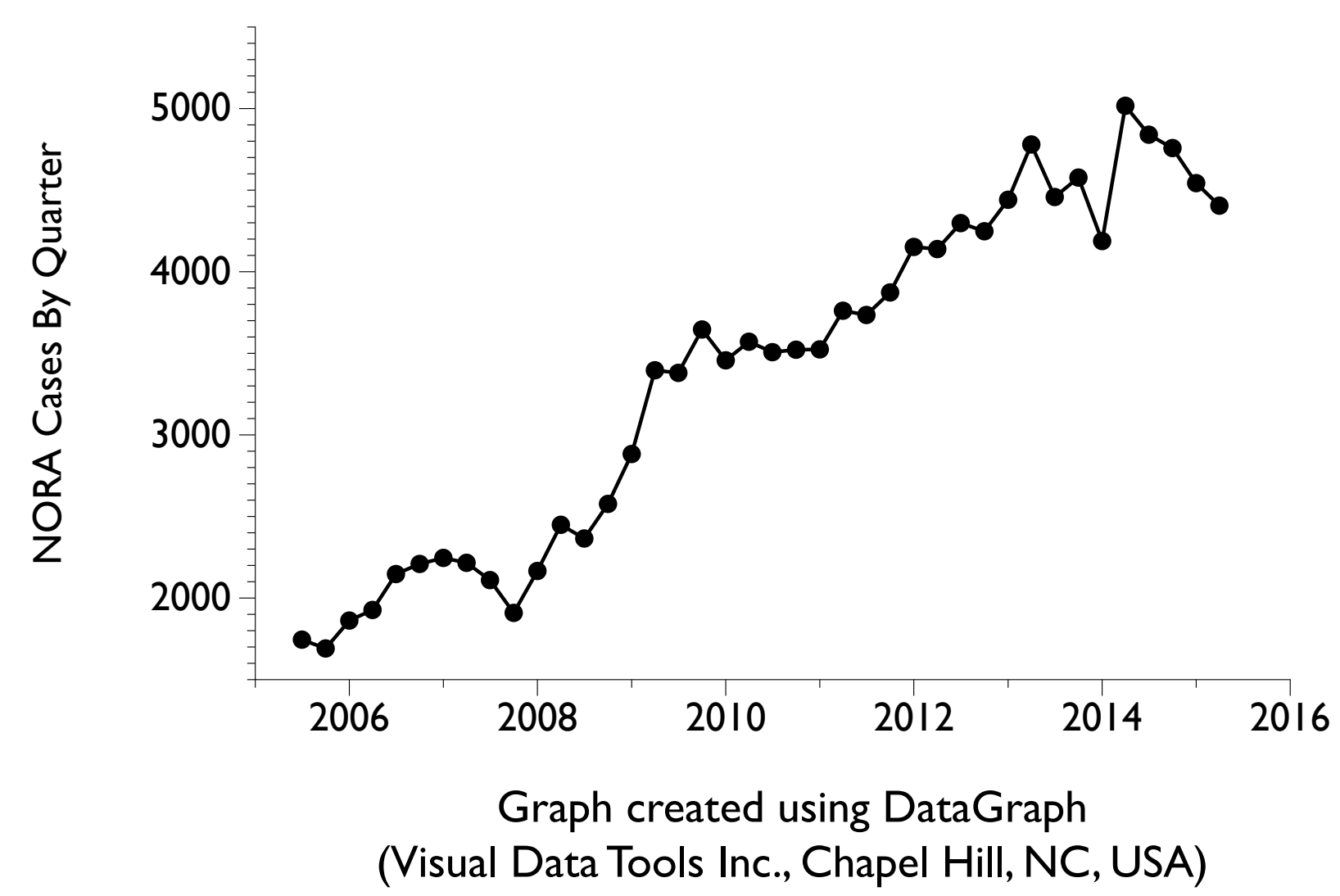

